\title{
Integration and instrument characterization of the cosmic infrared background experiment 2 (CIBER-2)
}

Chi H. Nguyen, Benjamin Stewart, Seung-Cheol Bang, James J. Bock, Asantha Cooray, et al.

Chi H. Nguyen, Benjamin Stewart, Seung-Cheol Bang, James J. Bock, Asantha Cooray, Kenta Danbayashi, Ambar DeSantiago, Viktor Hristov, Tomoya Kojima, Phillip Korngut, Kevin Kruse, Alicia Lanz, Dae-Hee Lee, Lunjun Liu, Jared Loewenthal, Peter Mason, Toshio Matsumoto, Shuji Matsuura, Ryo Ohta, Christian Pape, Won-Kee Park, Dorin Patru, James Parkus, Kei Sano, Aoi Takahashi, Mark Peryer, Kohji Takimoto, Kohji Tsumura, Takehiko Wada, Shiang-Yu Wang, Yasuhiro Yamada, Michael Zemcov, "Integration and instrument characterization of the cosmic infrared background experiment 2 (CIBER-2)," Proc. SPIE 10698, Space Telescopes and Instrumentation 2018: Optical, Infrared, and Millimeter Wave, 106984J (6 July 2018); doi: 10.1117/12.2311595

Event: SPIE Astronomical Telescopes + Instrumentation, 2018, Austin, Texas, United States 


\title{
Integration and Instrument Characterization of the Cosmic Infrared Background ExpeRiment 2 (CIBER-2)
}

Chi H. Nguyen ${ }^{a}$, Benjamin Stewart ${ }^{\mathrm{a}}$, Seung-Cheol Bang ${ }^{\mathrm{i}}$, James J. Bock ${ }^{\mathrm{b}, \mathrm{c}}$, Asantha Cooray ${ }^{\mathrm{d}}$, Kenta Danbayashie, Ambar DeSantiago ${ }^{a}$, Viktor Hristov ${ }^{b}$, Tomoya Kojima ${ }^{\mathrm{e}}$, Phillip Korngut ${ }^{\mathrm{b}}$, Kevin Kruse ${ }^{\mathrm{a}}$, Alicia Lanz ${ }^{\mathrm{b}}$, Dae-Hee Lee ${ }^{\mathrm{i}}$, Lunjun Liu ${ }^{\mathrm{b}}$, Jared Loewenthal ${ }^{\mathrm{a}}$, Peter Mason ${ }^{\mathrm{b}}$, Toshio Matsumoto ${ }^{\mathrm{f}, \mathrm{j}}$, Shuji Matsuura ${ }^{\mathrm{e}}$, Ryo Ohta ${ }^{\mathrm{e}}$, Christian Pape ${ }^{\mathrm{a}}$, Won-Kee Park ${ }^{\mathrm{i}}$, Dorin Patru $^{\mathrm{a}}$, James Parkus ${ }^{\mathrm{a}}$, Mark Peryer ${ }^{\mathrm{a}}$, Kei Sano ${ }^{\mathrm{e}}$, Aoi Takahashie, ${ }^{\mathrm{e}, \mathrm{g}}$, Kohji Takimoto ${ }^{\mathrm{e}}$, Kohji Tsumura $^{\mathrm{h}}$, Takehiko Wada ${ }^{\mathrm{f}}$, Shiang-Yu Wang ${ }^{\mathrm{j}}$, Yasuhiro Yamada ${ }^{\mathrm{e}}$, and Michael Zemcov ${ }^{\mathrm{a}, \mathrm{c}}$

${ }^{a}$ Center for Detectors, Rochester Institute of Technology, Rochester, New York 14623, USA;

${ }^{b}$ Department of Physics, Mathematics and Astronomy, California Institute of Technology, Pasadena, California 91125, USA;

${ }^{\mathrm{c} J e t}$ Propulsion Laboratory (JPL), National Aeronautics and Space Administration (NASA), Pasadena, California 91109, USA;

${ }^{\mathrm{d}}$ Department of Physics \& Astronomy, University of California, Irvine, California 92697, USA; eSchool of Science and Technology, Kwansei Gakuin University, Sanda, Hyogo 669-1337, Japan;

${ }^{\mathrm{f}}$ Institute of Space and Astronautical Science (ISAS), Japan Aerospace Exploration Agency (JAXA), Sagamihara, Kanagawa 252-5210, Japan;

${ }^{\mathrm{g}}$ Graduate University for Advanced Studies (SOKENDAI), Hayama, Kanagawa 240-0193, Japan;

${ }^{\mathrm{h}}$ Frontier Research Institute for Interdisciplinary Science, Tohoku University, Sendai, Miyagi 980-8578, Japan;

${ }^{i}$ Korea Astronomy and Space Science Institute (KASI), Daejeon 305-348, Republic of Korea; jInstitute of Astronomy and Astrophysics, Academia Sinica, Taipei 10617, Taiwan R. O. C.

\begin{abstract}
The extragalactic background light (EBL) is the integrated emission from all objects outside of the Milky Way galaxy. Imprinted by the history of stellar emission, the EBL in the near infrared traces light back to the birth of the first stars in the Universe and can allow tight constraints on structure formation models. Recent studies using data from the Spitzer Space Telescope and the first Cosmic Infrared Background ExpeRiment (CIBER-1) find that there are excess fluctuations in the EBL on large scales which have been attributed to either high redshift galaxies and quasars, or to stars that were stripped from their host galaxies during merging events. To help disentangle these two models, multi-wavelength data can be used to trace their distinctive spectral features. Following the success of CIBER-1, CIBER-2 is designed to identify the sources of the EBL excess fluctuations using data in six wavebands covering the optical and near infrared. The experiment consists of a cryogenic payload and is scheduled to launch four times on a recoverable sounding rocket. CIBER-2 has a 28.5 $\mathrm{cm}$ telescope coupled with an optics system to obtain wide-field images in six broad spectral bands between 0.5 and $2.5 \mu \mathrm{m}$ simultaneously. The experiment uses 2048 x 2048 HAWAII-2RG detector arrays and a cryogenic star tracker. A prototype of the cryogenic star tracker is under construction for a separate launch to verify its performance and star tracking algorithm. The mechanical, optical, and electrical components of the CIBER-2 experiment will have been integrated into the payload by mid-2018. Here we present the final design of CIBER-2 and our team's instrument characterization efforts. The design and analysis of the optical focus tests will be discussed. We also report on the performance of CIBER-2 support systems, including the cooling mechanisms and deployable components. Finally, we outline the remaining tasks required to prepare the payload for launch.
\end{abstract}

Send correspondence to C.H.N.: E-mail: chn2906@rit.edu, Telephone: 15854754102

Space Telescopes and Instrumentation 2018: Optical, Infrared, and Millimeter Wave, edited by Makenzie Lystrup, Howard A. MacEwen, Giovanni G. Fazio, Proc. of SPIE Vol. 10698, 106984J

(c) 2018 SPIE $\cdot$ CCC code: $0277-786 X / 18 / \$ 18 \cdot$ doi: $10.1117 / 12.2311595$ 
Keywords: Cosmology, Extragalactic astronomy, Rockets, Telescopes, Infrared background, Cryogenic infrared detectors, Cryogenic star trackers, Intensity mapping

\section{INTRODUCTION}

The extragalactic background light (EBL) is the integrated emission from all sources outside of the Milky Way Galaxy. More specifically, it is an ensemble of light from objects that cannot be resolved either due to their diffuse nature or due to the confused emission of the objects (e.g. galaxies at high redshift). The infrared (IR) portion of the EBL (also known as the Cosmic Infrared Background, CIB) is important because it contains contributions from the first generation of stars and galaxies formed during the Epoch of Reionization (EOR, $z>6$ ), ${ }^{1,2}$ the knowledge of which can inform and constrain cosmological models of structure formation. Unfortunately, this background is challenging to measure directly due to the presence of bright foregrounds like Zodiacal light (ZL), which can be up to 100 times brighter than the EBL. To investigate the EBL, instruments and observing techniques sensitive to its faint and diffuse nature are needed. ${ }^{3}$

Recently, intensity mapping using spatial fluctuations has been shown to be a useful probe of the EBL signal. $^{2,4-9}$ The EBL has a characteristic spectral feature known as the Lyman break, referring to the absence of rest frame ultra-violet photons due to absorption by the neutral intergalactic medium between the EOR and the observers. This break is observable around $\lambda \sim 1.0 \mu \mathrm{m}$ based on current models (for example 10) (Fig. 1). Taking advantage of this feature as well as the differing spatial structure of the EBL, we can distinguish it from foregrounds by performing intensity mapping at multiple wavebands. Studies using data from various telescopes like Spitzer, AKARI, and the first Cosmic Infrared Background ExpeRiment (CIBER-1) ${ }^{8,11-15}$ find that the EBL on large angular scales fluctuates more than expected from EOR models. Emission from stars stripped from their host galaxies during merger, also known as intra-halo light (IHL), has been proposed as one possible source for the excess fluctuations. ${ }^{8,9,16}$ To verify this scenario, CIBER-2 $2^{17,18}$ is currently being built based on the successful design of the previous experiment. Similar to CIBER-1, CIBER-2 comprises of a scientific payload, scheduled to launch multiple times on a sounding rocket provided by NASA. The scientific payload carries a telescope, imaging optics, and mechanical support systems. The first launch is projected for mid 2019.

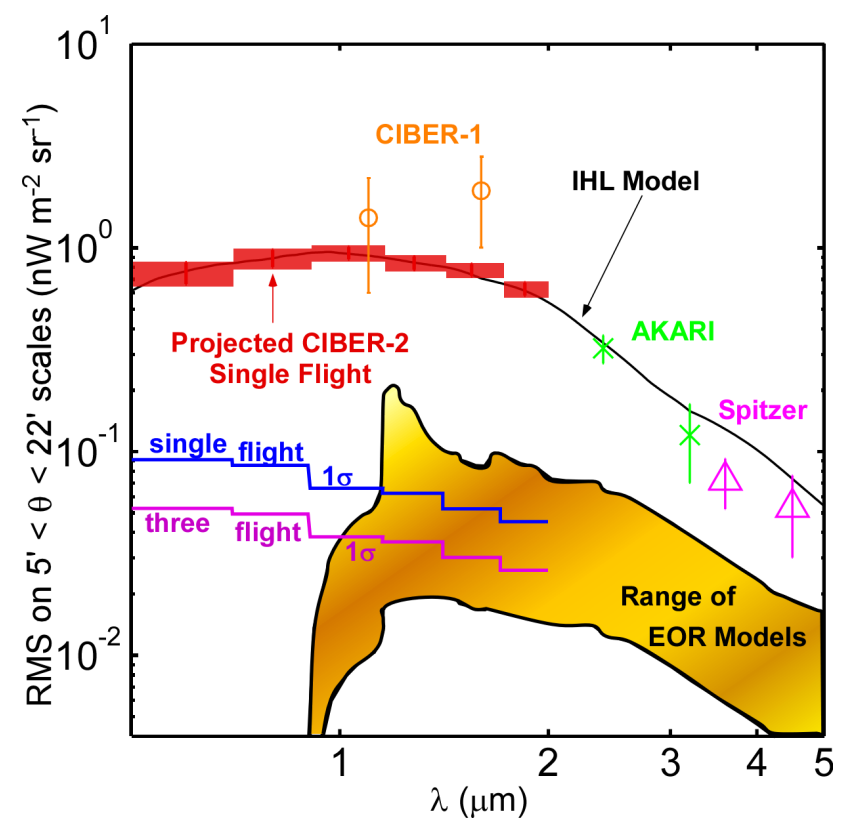

Figure 1. Theoretical model of the observed EBL. The best-fit model of the IHL shows that it has a different color than the EBL due to different redshift. It also does not have the Lyman break at IR/optical wavelength like EOR galaxies. The CIBER-2 science driver is to extend the spectral coverage into the optical window and increase the sensitivity to probe the Lyman break feature of the EOR and disentangle the IHL from the EBL. 
To achieve the instrument sensitivity needed to distinguish the EOR from the IHL (see Fig. 1), significant upgrades from the CIBER-1 design have been made to CIBER-2 (see 17,18). In this paper, we will report progress on the integration and instrument characterization of CIBER-2. Section 2 summarizes the finalized design of CIBER-2. Section 3 details the status of integration and the tests we have conducted on the payload. Finally, the next steps towards flight are outlined in Section 4.

\section{CIBER-2 OVERVIEW}

\subsection{Design and Flight Platform}

CIBER-2 will be launched on a Black Brant IX (BB IX) sounding rocket. The experiment will be integrated into the rocket at the NASA Wallops Flight Facility (WFF) in Virginia and launched from the White Sands Missile Range (WSMR) in New Mexico. Figure 2 shows the final mechanical model of the payload. Table 1 summarizes the features of CIBER-2. The designs of the mechanical structures, cryogenics, and optics are discussed in 17 and 18. Our final design does not deviate significantly from that reported in 17 and 18, so we refer the interested reader to those two articles for further details.

Sections 2.2 and 2.3 will focus on the cryogenic star tracker and the electronics which are new developments that were not covered in depth in previous publications.

Table 1. Summary of CIBER-2 features.

\begin{tabular}{|c|c|}
\hline Criteria & Description \\
\hline Sensitivity & $\begin{array}{l}\text { - Telescope: } \\
\text { - Ritchey-Chretién } \\
\text { - Primary aperture: } 28.5 \mathrm{~cm} \\
\text { - Field of view (FOV): } 2.3^{\circ} \times 2.3^{\circ}(\text { full }), 1.1^{\circ} \times 2.3^{\circ} \text { (filtered) }\end{array}$ \\
\hline Source masking & $\begin{array}{l}\text { - Deeper point source detection limits with advanced detector technology: } \\
\text { - Detectors: HAWAII- } 2 \mathrm{RG}^{\mathrm{TM}}(\mathrm{HgCdTe} \text { near infrared (NIR) })^{*} \\
\text { - Array size: } 2048 \times 2048 \\
\text { - Pixel size: } 18 \mu \mathrm{m} \times 18 \mu \mathrm{m} \\
\text { - Pixel scale: } 4 " \times 4 " \text { per pixel, half the pixel scale used in CIBER-1 } \\
\text { - Read noise: } 12 \mathrm{e}^{-}\end{array}$ \\
\hline Lyman break detection & $\begin{array}{l}\text { - Spectral coverage from optical into NIR: } \\
-3 \text { detectors coupled with telescope by lens optics and beamsplitters } \\
-6 \text { filters ( } 2 \text { per detector): } 0.60,0.80,1.03,1.28,1.55,1.85 \mu \mathrm{m} \\
\text { - Choose fields that have been observed by other instruments (e.g.: North } \\
\text { Ecliptic Pole) for more cross-correlated power spectra }\end{array}$ \\
\hline Airglow contamination & $\begin{array}{l}\text { - Sounding rocket platform (BB IX) to observe above Earth's atmosphere: } \\
\text { - Science observation at altitude }>250 \mathrm{~km} \\
\text { - } 4 \text { flights, } \sim 300 \text { s of data each }\end{array}$ \\
\hline Cryogenics to reduce noise & $\begin{array}{l}\text { - Cryostat (rocket skin) has a liquid nitrogen }\left(\mathrm{LN}_{2}\right) \text { tank, a radiation shield, } \\
\text { and a system of optical baffling to protect the telescope and optics from } \\
\text { thermal radiation from the rocket skin, door, and outside environment } \\
\text { - Operating at cold temperature }(77 \mathrm{~K}) \text { also lowers dark current noise in the } \\
\text { detectors }\end{array}$ \\
\hline Attitude control system & $\begin{array}{l}\text { - Standard star tracker provided by NASA } \\
\text { - Cryogenic star tracker using CIBER-2 telescope to correct for differential } \\
\text { thermal expansion of the rocket skin (see Section 2.2) }\end{array}$ \\
\hline
\end{tabular}

*HAWAII-xRG: HgCdTe Astronomy Wide Area Infrared Imager with Reference pixels and Guide mode. Number 2 refers to array size 2048 x 2048. Manufactured by Teledyne Technologies. 


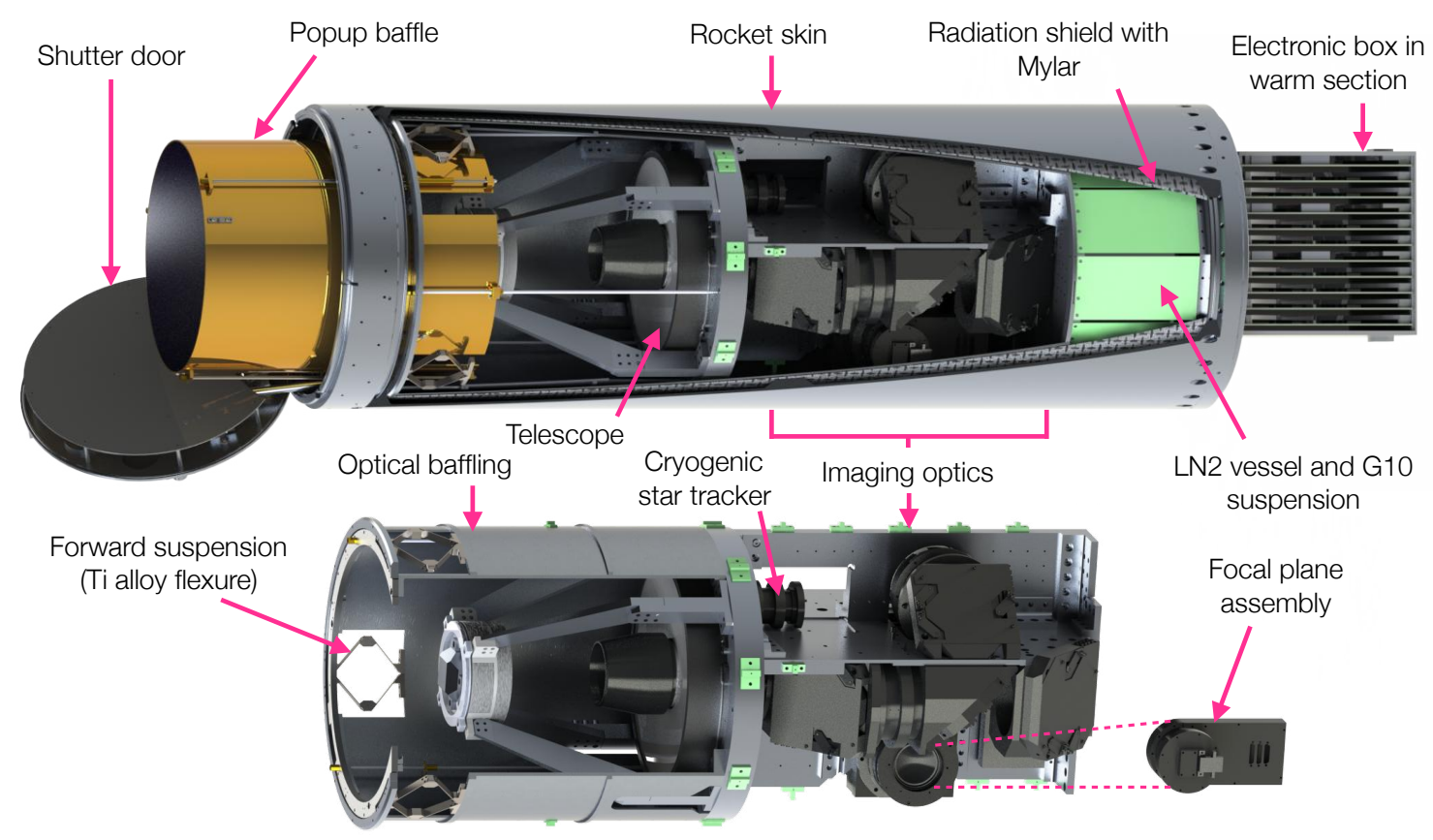

Figure 2. Solid model of the CIBER-2 payload.

\subsection{Cryogenic Star Tracking and Attitude Regulation System (CSTARS-1, CSTARS-2)}

BB IX rocket is equipped with a star tracker to update the pointing of the attitude control system. The star tracker monitors through a side-looking window, while our telescope observes from the aft of the payload section, along the longitudinal axis of the rocket. Therefore, there is an angle between the FOVs of our instrument and of the BB IX's star tracker. During flight, the BB IX skin is heated up due to friction with air molecules in the atmosphere, leading to a non-linear expansion of the rocket skin. When the rocket is in space, the skin cools down and contracts. As seen in CIBER-1 data, the gradual thermal contraction of the skin results in the angle between the FOVs of our instrument and of the rocket changing during the course of the science observation, resulting in a detectable drift in CIBER-1 sky images. While it is possible to correct for this pointing drift, the correction is not trivial and makes data analysis more difficult. Consequently, we want to avoid having to make this correction. To address the pointing drift issue, one solution is to have an additional star tracker looking in the same direction as the experiment. To do so, the star tracker needs to be housed in the same cryogenic section as the telescope and the imaging optics, sharing the same bore sight with the primary telescope. The addition of a cryogenic star tracker presents us with an opportunity to test a new scientific CMOS (sCMOS) detector in space. While sCMOS provides better performance in signal-to-noise ratio over the charge-coupled device (CCD) and conventional CMOS, an sCMOS application has not been verified in a cryogenic $(77 \mathrm{~K})$ payload. CIBER-2 suborbital flights can then provide empirical data on how well this kind of detector works at low temperature in space, which will prepare it for applications in future space missions.

A prototype of this star tracker is named the Cryogenic Star Tracking and Attitude Regulation System (CSTARS-1). CSTARS-1 is funded by a NASA Undergraduate Student Instrument Project (USIP) grant. Its firmware and tracking algorithm are developed by a team of interdisciplinary undergraduate students at the Rochester Institute of Technology (RIT). CSTARS-1 will fly on a separate BB IX mission to gather data that can verify the detector's hardware, firmware, and real-time star tracking algorithm. This payload includes a small lens telescope and an sCMOS detector assembly enclosed in a cryostat to keep the detector at $77 \mathrm{~K}$. On-board electronics will read the detector, identify stars and perform the tracking algorithm, then send the pointing information to the rocket's attitude control system. 
To integrate the star tracker into the CIBER-2 payload, we are building CSTARS-2 - an improved version of CSTARS-1. Table 2 lists the main components of both star trackers.

Table 2. CSTARS-1 and CSTARS-2 components.

\begin{tabular}{|c|c|c|}
\hline Components & CSTARS-1 & CSTARS-2 \\
\hline Telescope & $\begin{array}{l}\text { - Lens telescope, aperture: } 71.2 \mathrm{~mm} \\
- \text { FOV: } 6^{\circ} \times 6^{\circ}\end{array}$ & $\begin{array}{l}\text { - Use a pickoff mirror to share the CIBER-2 } \\
\text { telescope with the science cameras }\end{array}$ \\
\hline Detectors & $\begin{array}{l}\text { - STAR } 1000^{\dagger} \\
\text { - Array size: } 1024 \times 1024 \\
\text { - Pixel size: } 15 \mu \mathrm{m} \times 15 \mu \mathrm{m} \\
\text { - Pixel scale: } 21 " \times 21 " \text { per pixel } \\
\text { - Frame rate: } 5 \text { frames per second } \\
\text { - Readout mode: rolling shutter } \\
\text { - Read noise: } 35 \mathrm{e}^{-}\end{array}$ & $\begin{array}{l}\text { - Fairchild Imaging CIS2521F } \\
\text { - Array size: } 2560 \times 2160 \text { (total) } \\
2560 \times 1080 \text { (active) } \\
\text { - Pixel size: } 6.5 \mu \mathrm{m} \times 6.5 \mu \mathrm{m} \\
\text { - Pixel scale: } 10.5 " \times 10.5 " \text { per pixel } \\
\text { - Frame rate: } 10 \text { frames per second } \\
\text { - Readout mode: global shutter } \\
\text { - Read noise: } 2 \mathrm{e}^{-}\end{array}$ \\
\hline Cryogenics & $\begin{array}{l}\text { - Detector assembly is kept inside a } \\
\text { light-tight box, mounted directly on an } \mathrm{LN}_{2} \\
\text { tank, inside a vacuumed cryostat }\end{array}$ & $\begin{array}{l}\text { - Detector enclosure is mounted in the } \\
\text { optics section of CIBER-2 }\end{array}$ \\
\hline $\begin{array}{l}\text { Mechanical } \\
\text { support }\end{array}$ & - Cryostat mounted into a section of BB IX & $\begin{array}{l}\text { - Share the mechanical support of CIBER-2 } \\
\text { (cryostat, radiation shield, optical baffling) }\end{array}$ \\
\hline
\end{tabular}

\subsection{Electronics and Data Storage}

The data collected by CIBER-2 need to be stored on-board because they are transmitted at very high speed, $\sim 16$ times the capacity of the rocket to downlink. To carry the signal from the focal plane assembly in the cryogenic section to the warm electronics section, we are building cryogenic- and vacuum-rated cables that run inside and along the length of the rocket skin. Table 3 summarizes the main components of the system. Fig. 3 is a block representation of these components. The directions and types of communications between the different boards are also shown. Cryogenic components are located in the payload, and pass through the optics of CIBER-2. Warm and rocket electronics are in separate rocket sections that stay at room temperature.

The electronics of CIBER-2 can be divided into three main groups: the experiment electronics (blue and orange boards in Fig. 3), the cryogenic star tracker (green boards), and the rocket electronics (gray boards). The experiment and CSTARS-2 electronics are built by the CIBER-2 collaboration for data collection, while NASA provides the rocket electronics that control the rocket during flight and send telemetry data (speed, pointing, etc.) to the ground station. In order to synchronize the operation of the rocket and the experiment, an array processing house keeping (APHK) board is used to send clocking information from the rocket to the data acquisition system (DAS) board and the hard drive disk (HDD) storage boards. Each detector is read by a focal plane assembly (FPA) board, which then sends the analog signal to the DAS board for processing. The digitized signal is then forwarded to the HDD boards for storage. To accommodate the 32-channel readout of the detector, two DAS boards are used for each detector and two HDD boards are required to save three detectors' worth of data. For a detailed discussion of the data storage, see 19. The cryogenic star tracker electronics are separate from the experiment. The on-board processing unit (CSTARS-2 system) is adapted from the same electronics system used on CSTARS-1. It will read the sCMOS detector, run a star tracking algorithm in real-time, and then send the tracking information to the rocket attitude control system. The board will also send clocking, signals, and power from the rocket control to the sCMOS detector while forwarding telemetry data back to the attitude control system.

\footnotetext{
${ }^{\dagger}$ Manufactured by ON Semiconductor ${ }^{\circledR}$.

${ }^{\ddagger}$ Manufactured by Fairchild Imaging/BAE Systems.
} 
Table 3. CIBER-2 electronics components.

\begin{tabular}{|c|c|}
\hline Subsystem & Tasks \\
\hline $\begin{array}{l}\text { Focal plane assembly } \\
\text { (FPA) }\end{array}$ & - FPA board read the detector, 32-channel readout \\
\hline Cold harness cables & - Transmit signal from the FPB to the boards in the warm electronic section \\
\hline $\begin{array}{l}\text { Data acquisition system } \\
\text { (DAS) }\end{array}$ & $\begin{array}{l}\text { - Process analog-to-digital signal, } 16 \text { channels per board, } 2 \text { boards for each } \\
\text { detector }\end{array}$ \\
\hline $\begin{array}{l}\text { Array processing house } \\
\text { keeping (APHK) }\end{array}$ & $\begin{array}{l}\text { - Contain the Field-Programmable Gate Array (FPGA) which sends out } \\
\text { triggers for events, e.g: "test", "reset" } \\
\text { - Handle internal communication between the experiment and the rocket } \\
\text { control system, e.g: send clocking information to DAS boards, detector, etc. } \\
\text { - Provide power for the DAS boards and the data storage boards } \\
\text { - Relay changes in rocket pointing to DAS and HDD, e.g: on target, off target }\end{array}$ \\
\hline Hard disk drive (HDD) & - Store and protect data \\
\hline CSTARS-2 system & - Control the cryogenic star tracker \\
\hline Door cable & - Control the door (close/open) and the motor that deploys the popup baffle \\
\hline
\end{tabular}

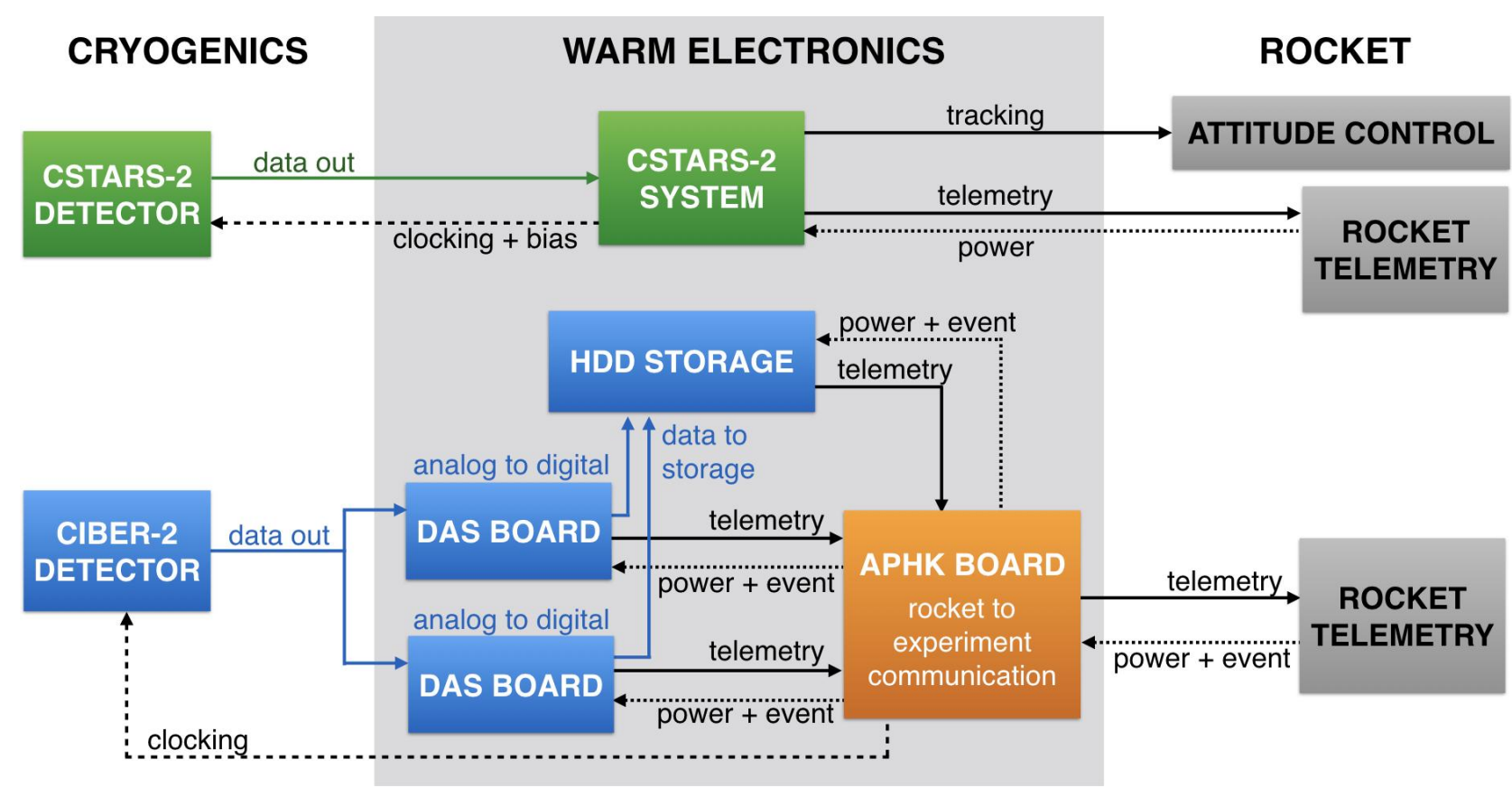

Figure 3. Schematics of CIBER-2 electronics, showing how the experiment electronics (blue and orange), the cryogenic star tracker (green), and the rocket electronics (gray) communicate with each other.

\section{CIBER-2 INTEGRATION AND CHARACTERIZATION}

CIBER-2 is currently in the integration and characterization stage. Most major components have been fabricated. After each component (mechanical, optical, electronic) is assembled, we verify that it is functional before we integrate it into the cryostat. As more systems are added into the cryostat, increasingly comprehensive evaluations are done to ensure that different components work together as a single system. In this section, we discuss the status of the CIBER-2 systems as well as how we evaluate them. 


\subsection{Mechanical Support Systems}

As of May 2018, most major support systems of CIBER-2 have been assembled into the cryostat in lab, including the rocket skin, the shutter door, the radiation shield, the $\mathrm{LN}_{2}$ tank, and the forward suspension flexures. As a test of the cryogenic system, the cryostat has been vacuumed and cooled down many times in 2017 and 2018. Thermometry installed inside the cryostat confirms that it can reach $77 \mathrm{~K}$ and maintain this temperature for the length of the lab tests. These cooling tests were made with no scientific instruments inside the cryostat (except the thermometers) so they can give us a baseline of how well the cryostat performs. In the next few months, we will add the optics and detectors into the cryostat and repeat the cooling test to verify that the cryostat still performs well when it is full of instruments.

While most mechanical supports have been validated, we are still waiting for the delivery of the pop-up and optical baffles. The delay in the baffles' fabrication is due to some modification in the designs. The original versions of the baffles as reported in 17 were such that the pop-up and optical baffles operated independent of each other. The optical baffle is a fixed structure that surrounds the telescope and shields it from thermal emission of the rocket skin. Meanwhile the pop-up baffle is an automated structure that extends beyond the aft of the rocket after the shutter door opens to shield the payload from stray IR emission (photons reflected off the shutter door, the rocket skin, or the Earth's atmosphere). Originally the pop-up baffle was designed to be deployed using a small motor mounted to the shutter door of the rocket, guided by a set of rods that move with it. However, after testing the prototype of the pop-up baffle in 2016, we discovered that this baffle needs an additional mechanical guide in order to maintain its alignment with the rocket's axis and to be deployed properly. To fix this issue, stationary guide rods for the pop-up baffle are added, using the optical baffle as mounting surfaces (see Fig. 4). The addition of the stationary rods combines the two baffles into a single system that must work together. The fabrication of the baffles began in late 2017. We expect them to be delivered in June 2018, after which point we will assemble them and commence testing of their operation.

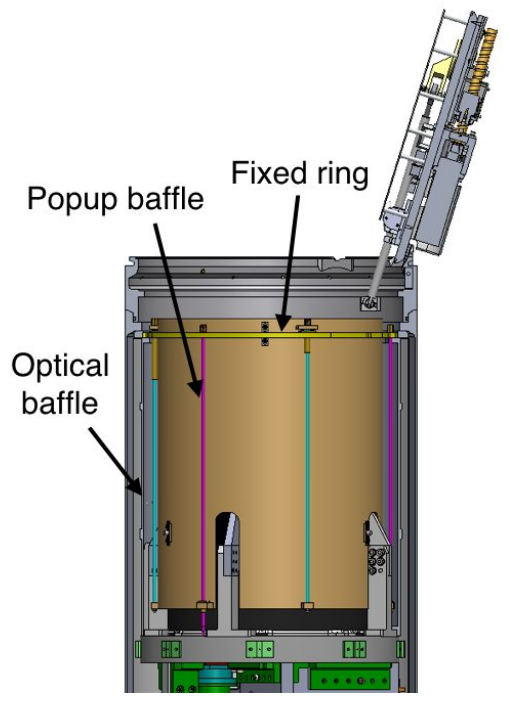

(a)

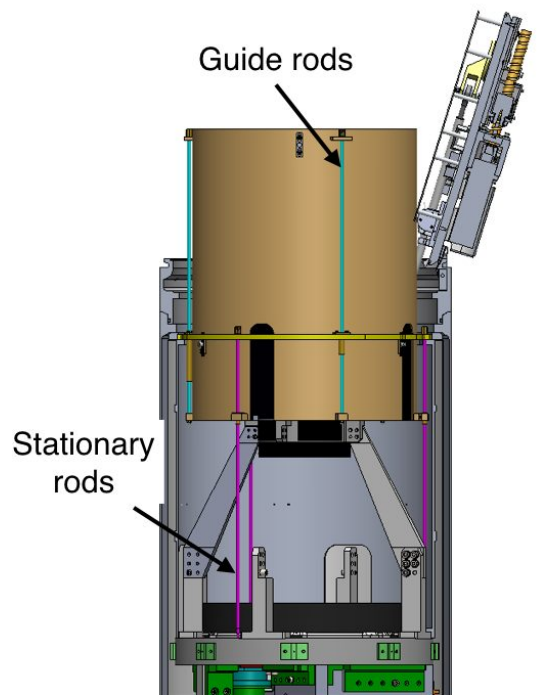

(b)

Figure 4. Current design of the pop-up and the optical baffles. (a) Pop-up baffle is stowed during launch and re-entry. (b) Pop-up baffle is deployed into an extended position for science observation.

In addition to the mechanical supports in the cryogenic section, we are working on an aluminum box to house and protect the warm electronics of CIBER-2. In addition to providing a mounting interface for the electronics boards, this electronics box has some metal ribs to support the boards against vibrations during launch and re-entry. To ensure that the box design is robust enough for this purpose, we test vibrated a dummy electronic board that was installed in a prototype of the box. We are analyzing the response of the board, the result of which will inform whether modifications to the box design are needed. 


\subsection{CIBER-2 Focal Plane Assemblies}

Mechanical mounting surfaces for the CIBER-2 detectors have been fabricated to the standards specified by the manufacturer. We also completed the filter assembly that will sit on top of each detector, the mechanical housing for the detector and its electronics, as well as the interface between the focal plane assembly and the lens optics. To verify the alignment and distance between the filters and the detectors, we are performing metrology on the filter's mechanical supports and the detector housings. After verification, the detectors and the filters will be installed into their housings and test images will be taken to validate the detectors' performance.

Fig. 5a shows the detector housing with the filter assembly stacked on top. The filters are on a quartz window which is located on top of the detector. When not in use, the quartz window is replaced with an aluminum plate (as seen in Fig. 5a) to protect the detector. Fig. 5b shows the mechanical box that houses the FPA board and highlights where the detector stack fits in.

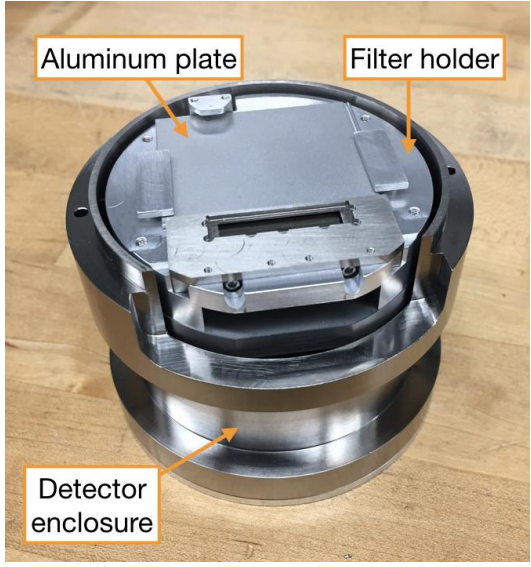

(a)

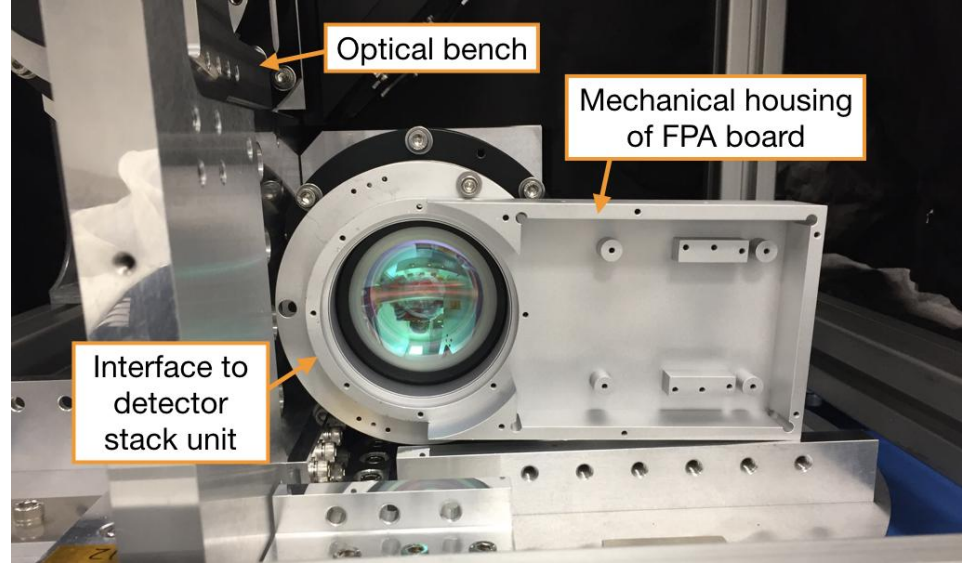

(b)

Figure 5. Photos of the CIBER-2 focal plane assembly. (a) One detector stack unit with the filter holder on top. When the unit is not in use, an aluminum plate replaces the quartz window to cover and protect the detector. (b) The housing of the FPA board, opened to show the posts that the board is mounted to. The detector stack fits into the circular cutout of this box.

\subsection{Lens Optics}

The lens optics components were built and delivered from Kwansei Gakuin University (KGU, Japan) to Caltech in late 2017. The optics assembly is divided into three arms, each corresponding to one detector assembly. The optics arms are labelled S, M, and L. The ray tracing model of these arms is presented in 18. Arms S and M have been assembled and characterization of these arms are in progress (Fig. 6). Arm L will be assembled in mid 2018.

To verify the alignment of the lens optics, we passed a light through each arm and took images of the light exiting the arm. To make the light signal uniform, we connected a light source to an integrating sphere before passing it through the lens optics. Significant coma was detected in the data taken for both arm S and arm M. Both arms share some common optics elements (Fig. 6), so they were taken apart from the optical bench and evaluated independently to trace the origin of the coma. The independent portions of arm S and arm M (those that come after the beam splitter) do not exhibit the large coma recorded previously, so we expect that the coma is due to their common optics elements.

One possible cause of the coma is that one (or more) lens in the common portion is tilted from its barrel due to the play between them. To check this, we documented the tilts of all lenses relative to their barrels by performing surface metrology. We subsequently identified very large tilts ( $\sim 2$ arcminutes or more) on some lenses. To fix these tilts, we attempted to cool some lens units down to $77 \mathrm{~K}$, at which point the barrels shrunk due to thermal contraction and the curvatures of the barrels pushed the lenses into alignment. The lenses were 
then warmed up to room temperature and we re-measured their tilts. One lens, G1, exhibited very large tilt initially ( $\sim 2.89$ arcminutes) and was shown to have better alignment after cooling (tilt $\sim 1.48$ arcminute). We are in the process of verifying if the coma issue is addressed by fixing the tilts.

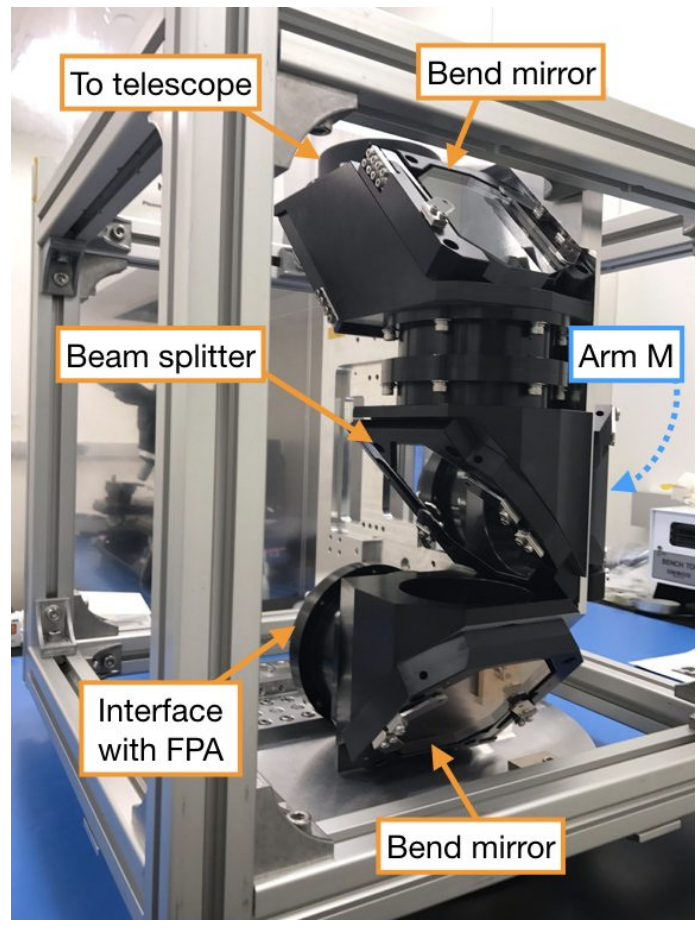

(a)

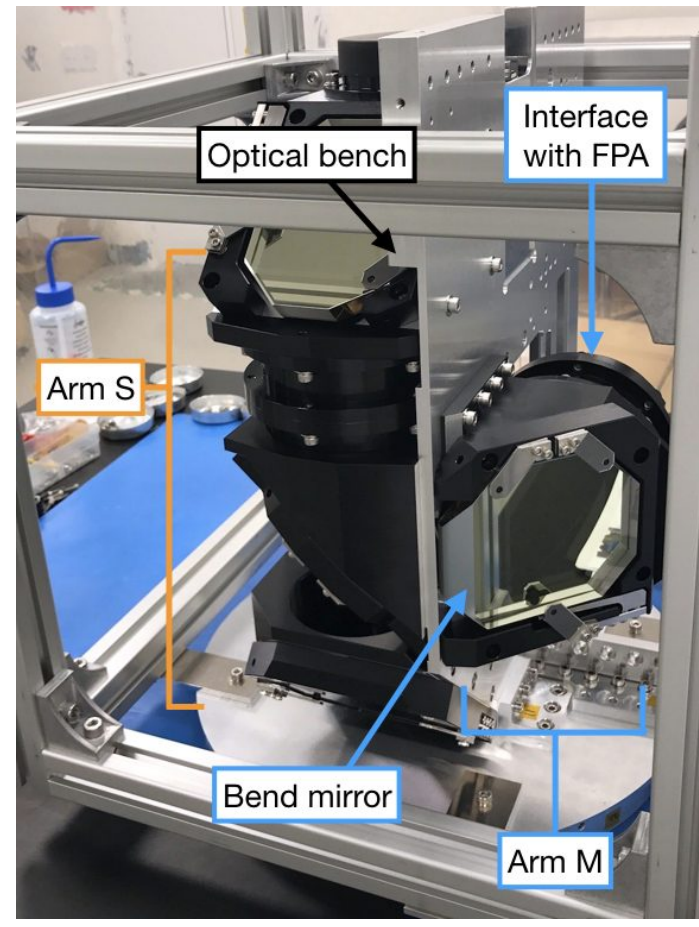

(b)

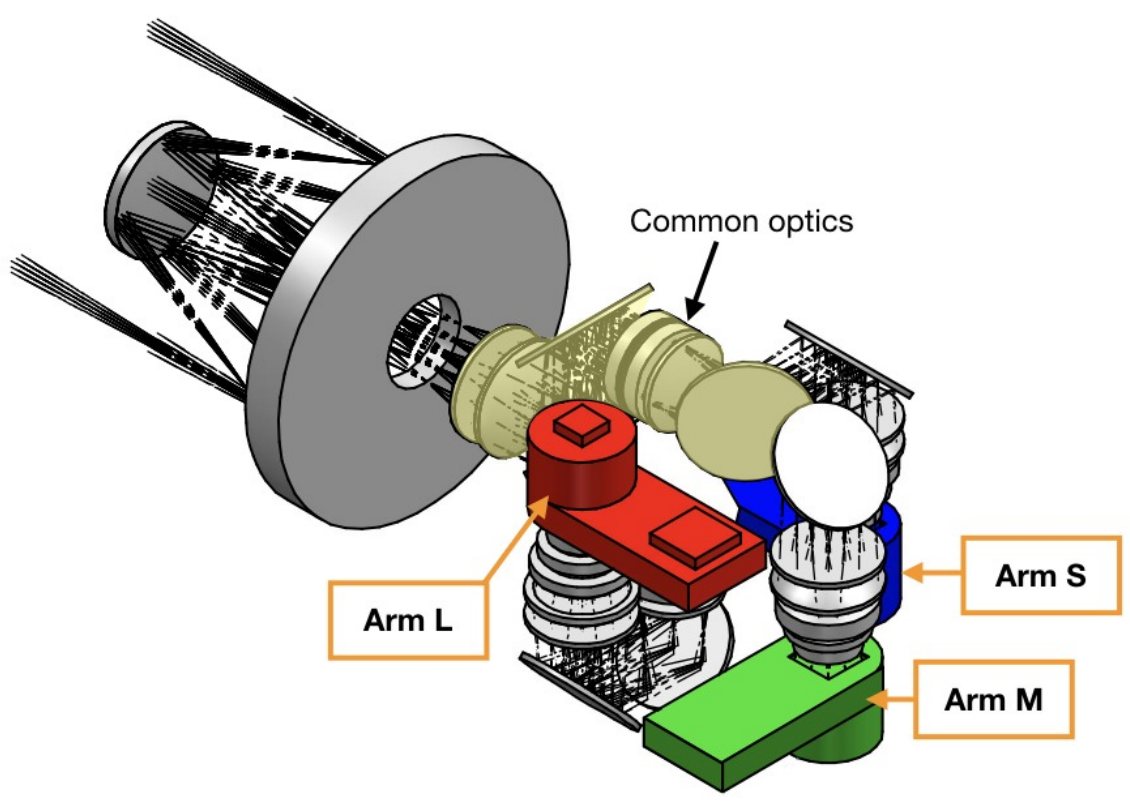

(c)

Figure 6. (a) Photo of arm S. (b) Photo of arm M, with arm S visible to the left. (c) Simplified ray tracing model of the lens optics, showing the common elements between arm S and arm M (highlighted in yellow). 


\subsection{Telescope}

The flight version of the telescope was delivered from KGU to Caltech in March 2018. In order to characterize the telescope, we have set up an apparatus in lab to measure the full width at half max (FWHM) of the point spread function (PSF) (see Fig. 7). We use a collimator to create parallel light rays from a pinhole, then take images of the pinhole using a camera located at the focus of the telescope. Fig. 8 is the image of a $2 \mu$ m pinhole mounted at the focus of the collimator.

In addition to verifying the focus of the telescope at room temperature, we also record data to help calibrate the telescope when it is at cryogenic. To do so, we vary the location of the pinhole and record how the images change to make a plot of pinhole offset from focus vs. PSF FWHM. When the telescope is integrated into the rocket skin and cooled down, we expect the focus of the telescope to shift slightly due to thermal contraction. The offset distance vs. PSF FWHM plot can then be used to find how much we need to change the pinhole location in order to counter the shift of the telescope and bring the image into focus again.

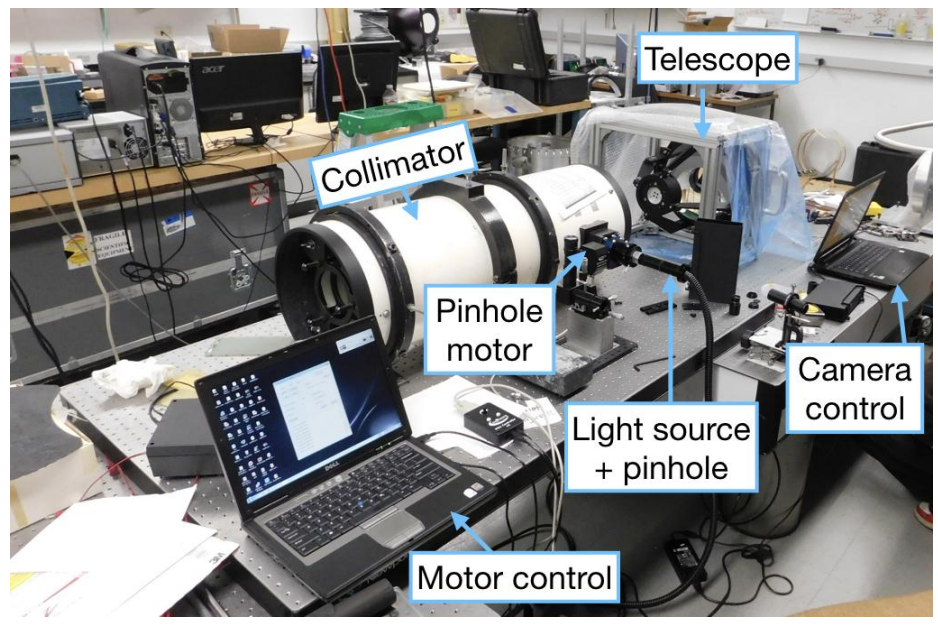

(a)

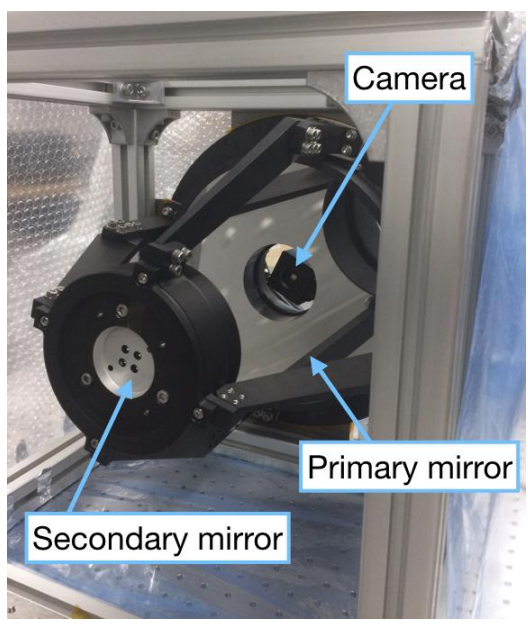

(b)

Figure 7. Setup for the telescope focus test at Caltech. (a) Light is passed through a pinhole to a collimator to create parallel rays that illuminate the telescope. A camera at the focus of the telescope is used to record images. A stepper motor is used to systematically and precisely vary the position of the pinhole. (b) Close-up view of the telescope and the camera.
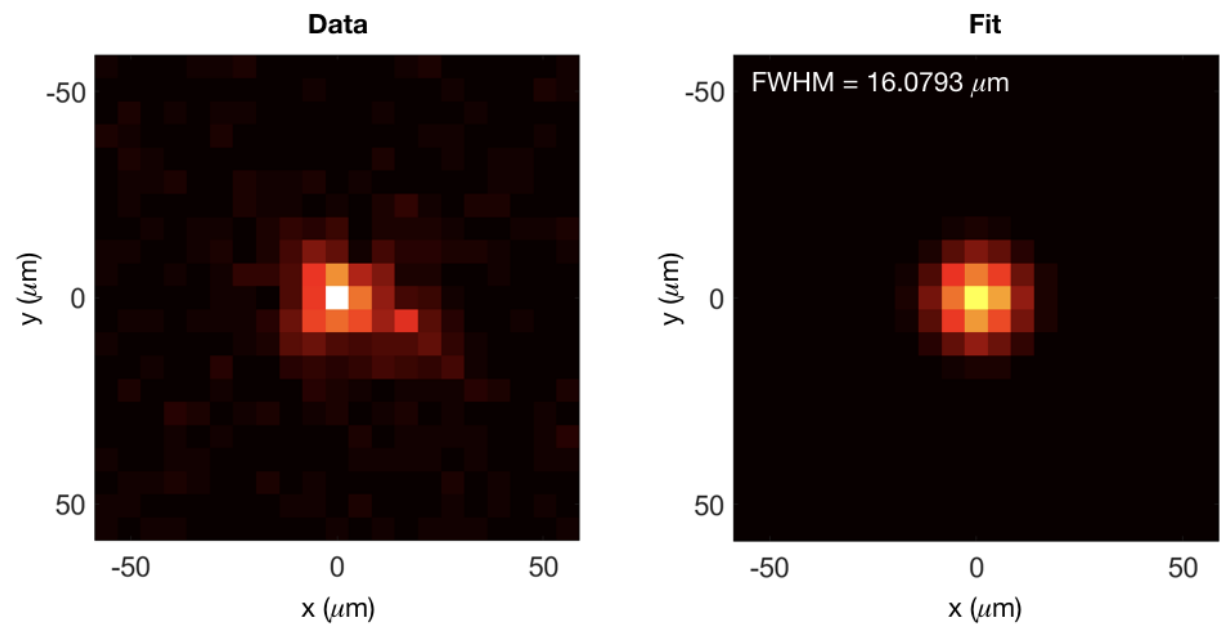

Figure 8. (Left) Image of a $2 \mu \mathrm{m}$ pinhole at the best focus of the telescope. (Right) Best fit of the data assuming a Gaussian PSF. 


\subsection{Electronics and Star Tracker}

\subsubsection{Experiment Electronics}

The APHK, DAS, and HDD boards of CIBER-2 have been fabricated and validated in lab. To successfully carry out the science observation, it is crucial that our electronics can coordinate seamlessly with the rocket control. In order to verify that our experiment electronics (DAS, APHK, HDD) conform to the electronics of the rocket provided by NASA, a handshake test with WFF hardware is being planned for mid 2018. In place of sky observation, ground support equipment has been constructed to simulate a stream of CIBER-2 data that we can then use to evaluate how well the CIBER-2 data acquisition and storage systems work.

\subsubsection{CSTARS-1}

CSTARS-1 mechanical and optical components were built in early 2017 (Fig. 9). The payload was integrated into a rocket section for a scheduled launch in May 2017, however due to unforeseeable technical difficulties the CSTARS-1 electronics were not completed and validated in time for this launch. By early 2018, we have solved most outstanding issues with the electronics and firmware, with only minor testing remaining to validate endto-end operation of the full system. Additionally, we have validated the star tracking algorithm using simulated stars in the lab, and will use a telescope mount to validate it on real stars in the summer of 2018. Finally, CSTARS-1 is waiting for an available BB IX launch to field test its payload and tracking algorithm in flight.

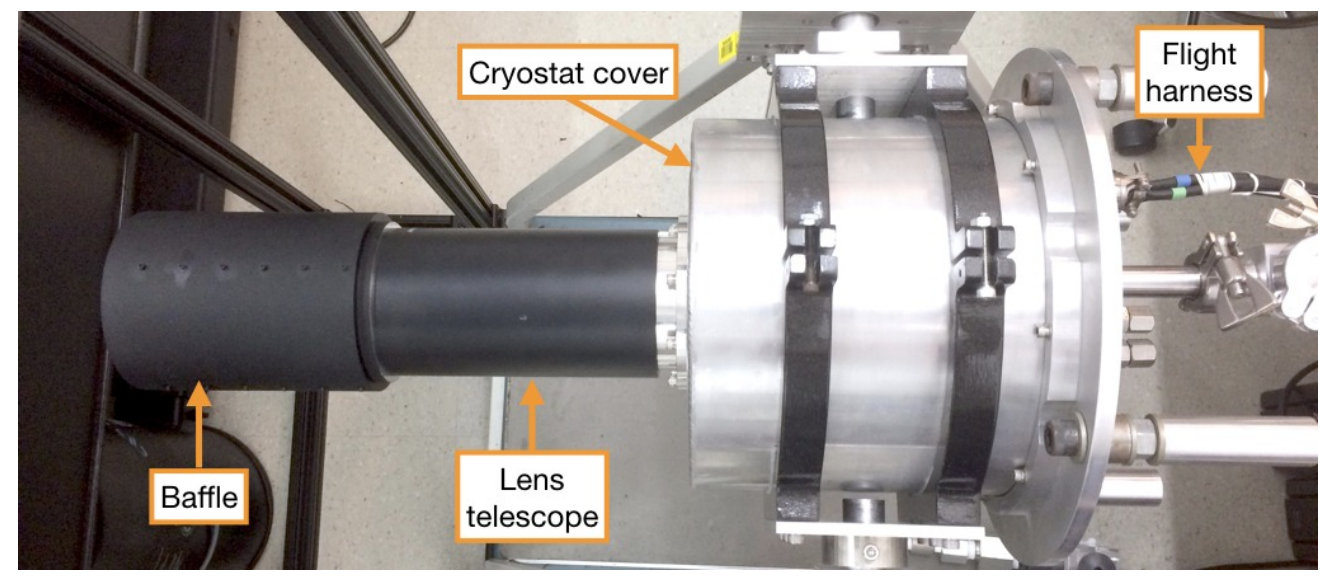

(a)

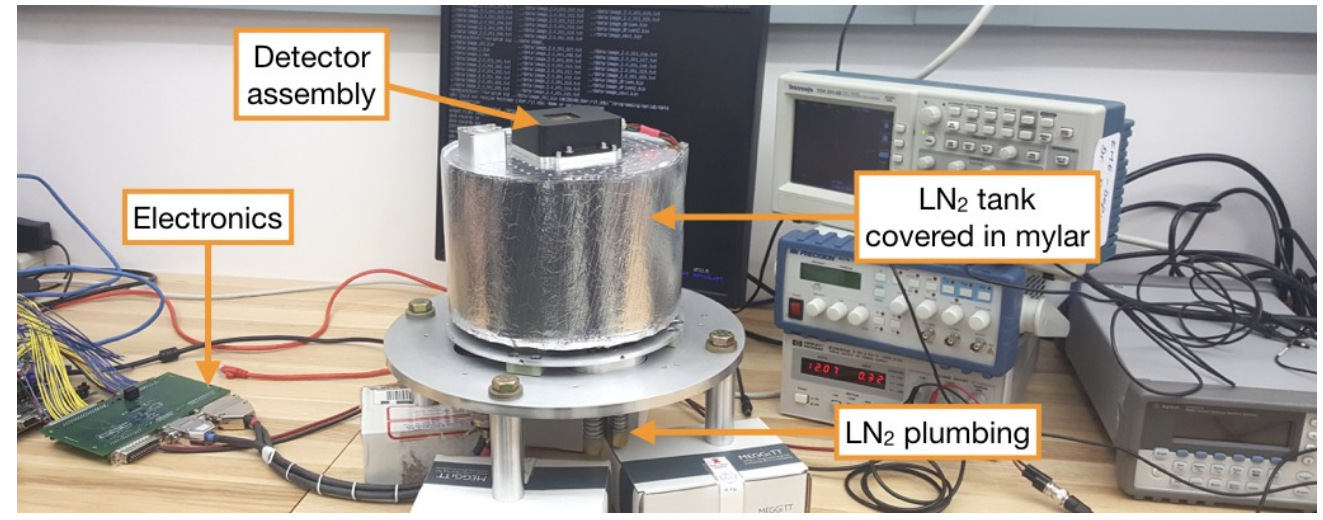

(b)

Figure 9. Progress of CSTARS. (a) The full assembly with the cryostat and the telescope. (b) The cryostat and the radiation shield are removed to show the detector assembly (the black box) and the $\mathrm{LN}_{2}$ tank covered in mylar. The detector is currently connected to one of the on-board computers (on the left) which is also connected to a display screen (in the background). 


\subsubsection{CSTARS-2}

CSTARS-2 is currently being validated and integrated into CIBER-2. The detector has been integrated into the focal plane board (Fig. 10a). The first revision of the electronics have been designed and are currently being used to characterize the detector (Fig. 10b). To meet the requirements of the experiment, the clocking and readout of the detector are undergoing final adjustments. In March 2018, the hardware interfacing CSTARS-2 with the attitude control system and the rocket telemetry encoder were validated at WFF, where a handshake between the CSTARS-2 hardware and a telemetry ground station was successfully established. A second revision of the interface electronics board is in fabrication for flight.

The mechanical enclosures of CSTARS-2 focal plane assembly and electronics board are also in fabrication. To ensure fit and alignment, the focal plane box will be fit check with the optics before getting hard black anodized. The flight harnessing has also been documented and assembly has begun.

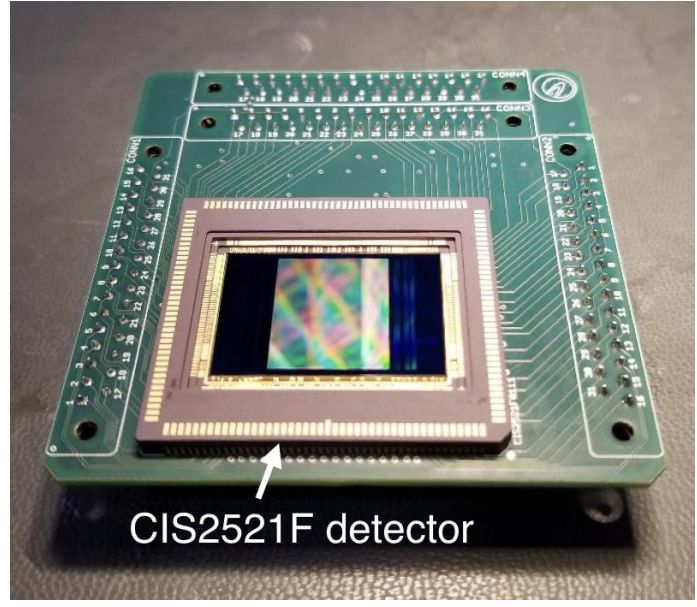

(a)

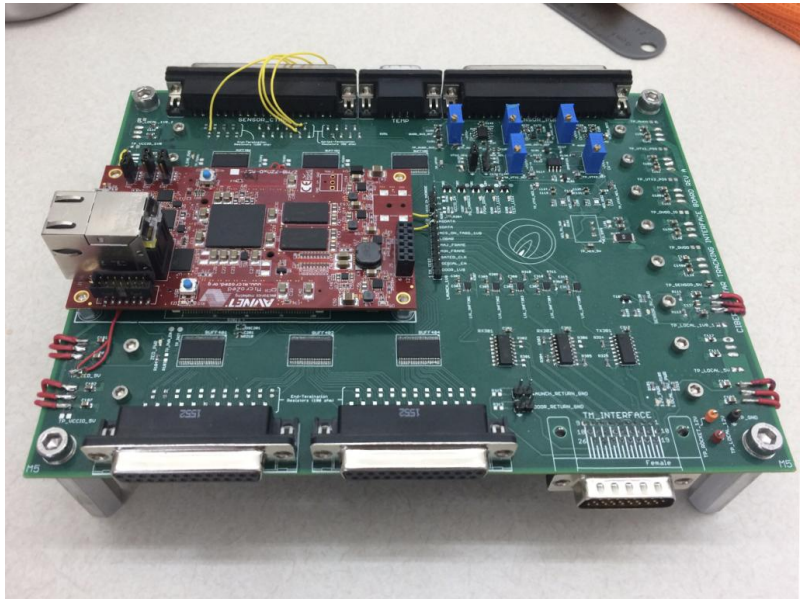

(b)

Figure 10. (a) CSTARS-2 focal plane assembly, showing the sCMOS detector. (b) CSTARS-2 interfacing board. The flight version of this board is currently being populated.

\section{FUTURE WORK}

Before integrating the CIBER-2 payload into the rocket at WFF, we need to completely assemble all components. The outstanding components include the baffles, arm L of the lens optics, and CSTARS-2 hardware. These parts also need to be validated and characterized after assembly.

We are working on tracing the origin of the coma in arm $\mathrm{S}$ and arm $\mathrm{M}$ of the lens optics. At the same time, we are setting up metrology and photogrammetry tests for the telescope to verify if the primary and secondary mirrors are aligned. If they are not aligned, we will need to fine-tune the secondary mirror's position so that we can obtain the smallest possible PSF FWHM.

After the focus test, we will proceed with integrating the HAWAII-2RG detectors into the optics assembly and characterize them. We will take images at room temperature and at $77 \mathrm{~K}$ to validate the performance of the detectors and the data acquisition, as well as to quantify the noise in the system. We will also obtain dark images to calibrate science data later.

CSTARS-2 hardware is expected to be delivered from RIT to Caltech in the third quarter of 2018. Before delivery, the detector must be validated at RIT with full confidence that the readout is consistent and accurate within the required parameters. The tracking algorithm and ground support software will be tested in parallel to ensure we can monitor the star tracking reliably in real-time. The electronics will be installed into its mechanical enclosure and checked for operation. A final handshake test of CSTARS-2 and the rocket interfaces will be performed at WFF. After that CSTARS-2 can be certified as fully functional and implemented into CIBER-2. 


\section{ACKNOWLEDGMENTS}

CIBER-2 was supported by NASA APRA research grants NNX07AI54G, NNG05WC18G, NNX07AG43G, NNX07AJ24G, and NNX10AE12G. Japanese participation was supported by KAKENHI (2034, 18204018, 19540250, 21340047, 21111004, and 15H05744) from Japan Society for the Promotion of Science (JSPS) and the Ministry of Education, Culture, Sports, Science and Technology (MEXT). Korean participation was supported by the Pioneer Project from Korea Astronomy and Space Science Institute (KASI). CSTARS was supported by USIP NASA grant NNX16AI82A. C.H.N was supported by NASA Headquarters under the NASA Earth and Space Science Fellowship Program - Grant 80NSSCK0706. We would like to thank the NASA Wallops Flight Facility engineers and staff for their technical supports.

\section{REFERENCES}

[1] Madau, P. and Silk, J., "Population III and the near-infrared background excess," Monthly Notices of the Royal Astronomical Society 359, L37-L41 (2005).

[2] Kashlinsky, A., Arendt, R. G., Mather, J., and Moseley, S. H., "Tracing the first stars with fluctuations of the cosmic infrared background," Nature 438, 45-50 (2005).

[3] Salvaterra, R., Burigana, C., Schneider, R., Choudhury, T. R., Ferrara, A., and Popa, L. A., "Cosmic radiative feedback from reionization .," Memorie della Societ Astronomica Italiana 80, 26 (2009).

[4] Cooray, A., Bock, J. J., Keatin, B., Lange, A. E., and Matsumoto, T., "First Star Signature in Infrared Background Anisotropies," The Astrophysical Journal 606, 611-624 (2004).

[5] Kashlinsky, A., Arendt, R. G., Mather, J., and Moseley, S. H., "New Measurements of Cosmic Infrared Background Fluctuations from Early Epochs," The Astrophysical Journal Letters 654, L5-L8 (2007).

[6] Thompson, R. I., Eisenstein, D., Fan, X., Rieke, M., and Kennicutt, R. C., "Evidence for a z $<8$ Origin of the Source-subtracted Near-Infrared Background," The Astrophysical Journal 666, 658-662 (2007).

[7] Matsumoto, T., Seo, H. J., Jeong, W.-S., Lee, H. M., Matsuura, S., Matsuhara, H., Oyabu, S., Pyo, J., and Wada, T., "AKARI Observation of the Fluctuation of the Near-infrared Background," The Astrophysical Journal 742, 124 (2011).

[8] Zemcov, M., Smidt, J., Arai, T., Bock, J., Cooray, A., Gong, Y., Kim, M. G., Korngut, K., Lam, A., Lee, D. H., Matsumoto, T., Matsuura, S., Nam, U. W., Roudier, G., Tsumura, K., and Wada, T., "On the Origin of Near-Infrared Extragalactic Background Light Anisotropy," Science 346, 6210 (2014).

[9] Cooray, A., Smidt, J., de Bernardis, F., Gong, Y., Stern, D., Ashby, M. L. N., Eisenhardt, P. R., Frazer, C. C., Gonzalez, A. H., Kochanek, C. S., Kozłowski, S., and Wright, E. L., "Near-infrared background anisotropies from diffuse intrahalo light of galaxies," Nature 490, 514-516 (2012).

[10] Robertson, B. E., Ellis, R. S., Furlanetto, S. R., and Dunlop, J. S., "Cosmic Reionization and Early Starforming Galaxies: A Joint Analysis of New Constraints from Planck and the Hubble Space Telescope," The Astrophysical Journal Letters 802, L19 (2015).

[11] Bock, J., Battle, J., Cooray, A., Kawada, M., Keating, B., Lange, A., Lee, D.-H., Matsumoto, T., Matsuura, S., Pak, S., Renbarger, T., Sullivan, I., Tsumura, K., Wada, T., and Watabe, T., "The cosmic infrared background experiment," New Astronomy Reviews 50, 215-220 (2006).

[12] Zemcov, M., Arai, T., Battle, J., Bock, J., Cooray, A., Hristov, V., Keating, B., Kim, M. G., Lee, D. H., Levenson, L. R., Mason, P., Matsumoto, T., Matsuura, S., Nam, U. W., Renbarger, T., Sullivan, I., Suzuki, K., Tsumura, K., and Wada, T., "The Cosmic Infrared Background Experiment (CIBER): A Sounding Rocket Payload to Study the near Infrared Extragalactic Background Light," The Astrophysical Journal Supplement 207, 31 (2013).

[13] Bock, J., Sullivan, I., Arai, T., Battle, J., Cooray, A., Hristov, V., Keating, B., Kim, M. G., Lam, A. C., Lee, D. H., Levenson, L. R., Mason, P., Matsumoto, T., Matsuura, S., Mitchell-Wynne, K., Nam, U. W., Renbarger, T., Smidt, J., Suzuki, K., Tsumura, K., Wada, T., and Zemcov, M., "The Cosmic Infrared Background Experiment (CIBER): The Wide-field Imagers," The Astrophysical Journal Supplement 207, $32(2013)$. 
[14] Korngut, P. M., Renbarger, T., Arai, T., Battle, J., Bock, J., Brown, S. W., Cooray, A., Hristov, V., Keating, B., Kim, M. G., Lanz, A., Lee, D. H., Levenson, L. R., Lykke, K. R., Mason, P., Matsumoto, T., Matsuura, S., Nam, U. W., Shultz, B., Smith, A. W., Sullivan, I., Tsumura, K., Wada, T., and Zemcov, M., "The Cosmic Infrared Background Experiment (CIBER): The Narrow-Band Spectrometer," The Astrophysical Journal Supplement 207, 34 (2013).

[15] Tsumura, K., Matsumoto, T., Matsuura, S., Sakon, I., and Wada, T., "Low-Resolution Spectrum of the Extragalactic Background Light with the AKARI InfraRed Camera," Publications of the Astronomical Society of Japan 65, 121 (2013).

[16] Matsuura, S., Arai, T., Bock, J. J., Cooray, A., Korngut, P. M., Kim, M. G., Lee, H. M., Lee, D. H., Levenson, L. R., Matsumoto, T., Onishi, Y., Shirahata, M., Tsumura, K., Wada, T., and Zemcov, M., "New Spectral Evidence of an Unaccounted Component of the Near-infrared Extragalactic Background Light from the CIBER," The Astrophysical Journal 839, 7 (2017).

[17] Lanz, A., Arai, T., Battle, J., Bock, J., Cooray, A., Hristov, V., Korngut, P., Lee, D. H., Mason, P., Matsumoto, T., Matsuura, S., Morford, T., Onishi, Y., Shirahata, M., Tsumura, K., Wada, T., and Zemcov, M., "Studying extragalactic background fluctuations with the Cosmic Infrared Background ExpeRiment 2 (CIBER-2)," in [Society of Photo-Optical Instrumentation Engineers (SPIE) Conference Series], Society of Photo-Optical Instrumentation Engineers (SPIE) Conference Series 9143, 3 (2014).

[18] Shirahata, M., Arai, T., Battle, J., Bock, J., Cooray, A., Enokuchi, A., Hristov, V., Kanai, Y., Kim, M. G., Korngut, P., Lanz, A., Lee, D.-H., Mason, P., Matsumoto, T., Matsuura, S., Morford, T., Ohnishi, Y., Park, W.-K., Sano, K., Takeyama, N., Tsumura, K., Wada, T., Wang, S.-Y., and Zemcov, M., "The cosmic infrared background experiment-2 (CIBER-2) for studying the near-infrared extragalactic background light," in [Space Telescopes and Instrumentation 2016: Optical, Infrared, and Millimeter Wave], Proceedings of SPIE 9904, 99044J (2016).

[19] Park, W.-K., Bang, S.-C., Battle, J., Bock, J. J., Cooray, A., Danbayashi, K., DeSantiago, A., Hristov, V., Kojima, T., Korngut, P., Lanz, A., Lee, D.-H., Liu, L., Loewenthal, J., Peter Mason, P., Matsumoto, T., Matsuura, S., Nguyen, C. H., Ohta, R., Pape, C., Patru, D., Parkus, J., Peyer, M., Sano, K., Stewart, B., Takahashi, A., Takimoto, K., Tsumura, K., Wada, T., Wang, S.-Y., Yamada, Y., and Zemcov, M., "Development of data storage system and GSE for cosmic infrared background experiment 2 (CIBER-2," in [Space Telescopes and Instrumentation 2018: Optical, Infrared, and Millimeter Wave], Proceedings of SPIE 10698 (2018). 